\title{
Efecto de las nuevas tecnologías en el Perú
}

\author{
Effect of new technologies in Peru \\ Efeito das novas tecnologias no Peru
}

\section{ARTÍCULO GENERAL}

\section{César Loo Gil \\ cesarloo@biofab.com.pe \\ https://orcid.org/0000-0001-8396-5972}

Científico Investigador de BioFab Inc. y del Centro de Investigación \& Producción Científica IDEOs, Lima - Perú

Recibido 05 de Febrero 2021 | Arbitrado y aceptado 19 de Abril 2021 | Publicado 28 de Julio 2021

\section{RESUMEN}

La Ciencia y Tecnología albergan una interacción teórica y práctica que en grupo son clave para el desarrollo nacional, el aumento y sostenibilidad económica, la formación de expertos y la generación de competencias, conforme a las necesidades del mercado. Examinar los primordiales impactos y retos que las novedosas tecnologías de información y comunicación plantean al sector tecnológico y a la sociedad en su grupo, es el objetivo de este análisis, en un escenario donde la creciente fusión y desarrollo de la informática y las telecomunicaciones brota como el elemento primordial que impulsa la globalización a pasos agigantados. La generación de riqueza, la construcción de trabajo calificado, la optimización de la productividad, la igualdad económica y el desarrollo de nuevos servicios, se tienen que cada vez más, a la contribución de las novedosas tecnologías y los servicios de telecomunicaciones en las múltiples esferas de la sociedad y la economía.

Palabras claves: Desarrollo, Ciencia, Tecnología, Perú

\section{ABSTRACT}

Science and Technology house a theoretical and practical interaction that as a group are key for national development, economic growth and sustainability, the training of experts and the generation of skills, according to the needs of the market. Examining the main impacts and challenges that the new information and communication technologies pose to the technology sector and to society in its group is the objective of this analysis, in a scenario where the growing fusion and development of information technology and telecommunications sprouts as the essential element that drives globalization by leaps and bounds. The generation of wealth, the construction of qualified work, the optimization of productivity, economic equality and the development of new services, are increasingly due to the contribution of new technologies and telecommunications services in multiple spheres of society and the economy.

Keywords: Development, Science, Technology, Peru

\section{RESUMO}

Ciência e Tecnologia abrigam uma interação teórica e prática que, em conjunto, são fundamentais para o desenvolvimento nacional, o crescimento econômico e a sustentabilidade, a formação de especialistas e a geração de competências, de acordo com as necessidades do mercado. Analisar os principais impactos e desafios que as novas tecnologias da informação e comunicação colocam ao setor da tecnologia e à sociedade do seu grupo é o objetivo desta análise, num cenário onde a crescente fusão $\mathrm{e}$ desenvolvimento das tecnologias da informação e das telecomunicações desponta como elemento essencial que impulsiona a globalização aos trancos e barrancos. A geração de riqueza, a construção de trabalho qualificado, a otimização da produtividade, a igualdade económica e o desenvolvimento de novos serviços, devem-se cada vez mais ao contributo das novas tecnologias e dos serviços de telecomunicações nas múltiplas esferas da sociedade e da economia.

Palavras-chave: Desenvolvimento, Ciência, Tecnologia, Peru 
Introducción

El siguiente artículo comprende los principales efectos que han generado las nuevas tecnologías en el Perú, marcando un principio de Conceptos Básicos para una mejor comprensión, los insuficientes incentivos tecnológicos, la labor de la institución Concytec y el sistema de Desarrollo Nacional para la Ciencia y Tecnología. El objetivo que buscamos lograr en los lectores es informar sobre la existencia o no del mencionado desarrollo respaldado de autores expertos en los campos de innovación científica y tecnológica adecuado a la realidad nacional.

En primera instancia - implícito en esta convergencia -, los sectores y tecnologías que intervienen en este proceso no deberían ser más analizados por separado, en el tamaño que no únicamente desaparecen las fronteras entre ellos, sino que, en su evolución, otorgan origen a nuevos mercados, productos y servicios.

Después, se debe resaltar la ausencia, por ahora, de un sistema de estadísticas sucesivas y confiables de la evolución de los primordiales indicadores. El motivo, se apunta en un párrafo anterior, es que la transformación del sector tecnológico de las comunicaciones y la informática fue tan instantánea que las naciones, incluyendo el Perú, no estuvieron preparados para medir y cuantificar los cambios. Estas carencias, con las diferencias del caso, además la poseen organismos de la talla de la OECD, UIT, la Comisión Europea y el Departamento de Negocio de los EE. UU., que trabajan en la actualidad por subsanar estas deficiencias.

Otro aspecto fundamental es la complejidad para conceptualizar varias cambiantes.

Ejemplificando, ¿qué es un cliente de Internet? Es acaso el que navega, 10, 20 o más horas al mes, o el que acceso por lo menos una vez a la semana o al mes, o cualquier persona que se conecte aun cuando sea rara vez. Lo mismo ocurre con el número de host, portales, páginas web, visitas llevadas a cabo, perfil de los usuarios, cómo son utilizados y notados los nuevos servicios, entre otros aspectos. Cada territorio, e inclusive cada organización, que examina el mercado, lo mide a su criterio, con sus propias definiciones y limitaciones, de ahí que las mediciones tienen que considerarse más como herramientas que brindan un orden de intensidad y una dirección, que una cifra rigurosa y precisa.

\section{Metodología}

La metodología que se utilizó consta de una búsqueda de información a base de papers, revistas, análisis de aplicaciones actuales y páginas web especializadas para entender su funcionamiento, el nivel de desarrollo obtenido en la actualidad y cuáles son sus principales aplicaciones. Además, se han empleado análisis de modelos estratégicos 
acordes para analizar el atractivo de las industrias en la adopción de la tecnología y así determinar a través de modelos de análisis de negocio cuáles son las industrias en que tendrá mayor incidencia. La búsqueda de la información para la revisión de literatura se hizo consultando las siguientes bases de datos como son, Scopus, Proquest y Google Académico para encontrar revistas del más alto nivel científico. Asimismo, cabe mencionar que para la realización de lo mencionado se emplearon también palabras clave como Desarrollo, Ciencia, Tecnología, Perú.

Cada uno de estos descriptores se combinaron entre sí durante la búsqueda utilizando los operadores boléanos "and" y “or". Además, algunos de los criterios o filtros que se utilizaron para hacer muchos más específica la búsqueda y ser más precisos con los documentos encontrados en las diferentes bases de datos consultadas fueron: que sean artículos de revistas, y la antigüedad de la publicación que no sea mayor a 5años.Toda la información previa relacionada a los filtros aplicados y las especificaciones sirvieron como criterios de inclusión, es decir que sean artículos publicados en revistas científicas, que no excedan los cinco años de antigüedad, que la temática guarde relación con las variables de creatividad artística y tiempos de pandemia. Se excluyeron, en tanto, todos los documentos que no respetaran lo postulado, así como aquellos que estaban incompletos o que tenían enlaces averiados.

Resultados y discusión

\subsection{Nociones básicas}

Relación ciencia y tecnología

Una primera aproximación para una mejor comprensión del problema a ser discutido, es la interacción entre ciencia y tecnología como elemento de desarrollo en cualquier sociedad.

Según Bunge (2014) la ciencia “es el razonamiento sistemático, preciso, verificable y por ende falible, la gente amasa y remoldea la naturaleza, sometiéndola a sus necesidades". Según el creador, la ciencia tiene métodos regulados, los cuales tienen la posibilidad de ser revisados y falibles. Es aquí donde los individuos intervienen para adecuar los métodos según sus necesidades de conocimientos para solucionar inconvenientes que le inquietan. 
Bunge (2014) dice que la ciencia "es el análisis de las interacciones empíricamente demostrables, o sea, la construcción del entendimiento desde realidades objetivas por la experiencia". Aquí da comprender que su criterio sobre ciencia trata una compresión de hechos reales basados en la observación y vivencia, de tal forma, y grupo al primer criterio, se puede conceptualizar a la ciencia como el grupo de métodos reales con base a la vivencia y observación de hechos para cubrir las necesidades de los individuos.

La tecnología, sin embargo, según Bunge (1985) “es el vastísimo campo de indagación, diseño y idealización que usa conocimientos científicos con la intención de mantener el control de cosas o procesos naturales, de diseñar instrumentos o procesos, o de concebir operaciones de forma racional".Lo cual supone que la gente realiza la función de ejercer conocimientos, técnicas, procesos, medios y grupos necesarios para producir bienes y servicios, a fin de crear herramientas como resultados de este grupo de medios.

Al final, Bunge (1985) incorpora que la tecnología "se muestra como una simbiosis entre el entendimiento teórico de la ciencia, cuya finalidad es la averiguación de la realidad, con la técnica, cuya finalidad es la utilidad. El objetivo de la tecnología podría ser la averiguación de una verdad útil”.

Tanto la ciencia como la tecnología albergan una interacción estrecha, cuya finalidad es la deducción de un realizado real, usando en el proceso de trabajo las técnicas teóricas y prácticas primordiales para una compresión descriptiva de los inconvenientes de la verdad y planteamientos de resoluciones viables.

Insuficientes incentivos para el desarrollo de la Ciencia y Tecnología

En nuestra región los conceptos de ciencia y tecnología no se complementa con la verdad de la formación escolar, quienes carecen de los recursos necesarios que abordaremos luego.

Insuficientes incentivos económicos

Según con Vela, Acevedo, Yesquen y Venturra (2018), "existen insuficientes incentivos para la ciencia y tecnología, y esto se explica por insuficientes fuentes de financiamiento, insuficientes aparatos de promoción para la absorción, transferencia y difusión tecnológica, inadecuadas fuentes de financiamiento $\mathrm{e}$ inadecuado apoyo del emprendimiento tecnológico".

Los autores aseguran una penosa realidad que probablemente no tenga solución, los incentivos económicos para la ciencia y tecnología en nuestra región son escasos, o sencillamente inexistentes. Y más todavía, podríamos dialogar de la corrupción una vez que refieren el concepto "inadecuadas", el cual da a dar por sentado que si bien existe el 
incentivo económico, solo rebasa la realidad de dichos medios. Además de eso, se habla de una escasa transmisión de los temas, grupo a que si existe un aumento tecnológico en nuestra región, no está gestionado como es debido.

Falta de Investigadores y personal calificado

Desde un aspecto regional, Vela, Acevedo, Yesquen y Venturra (2018) señalan que "en Latinoamérica aún existen retos pendientes en cuanto a investigación e innovación, pues dichos desafíos serían considerados instrumentos fundamentales para luchar contra la pobreza, mejorar en los índices de salud, alcanzando un desarrollo en los países latinoamericanos de manera sostenible, inclusiva, integrada y equitativa".

Lo que significa que la situación n o solo es característico en nuestro país, sino también de nuestra región y aún existen retos pendientes de investigación, relacionados a la ciencia, e innovación y tecnología. La importancia de darle el valor necesario a estos pilares del desarrollo humano, y como señalan los autores, seria instrumentos claves para combatir la pobreza y los deficientes sistemas de atención médica, que llevaría al desarrollo de toda la región de forma paralela.

Para Seclén (2017) “más que tratar de crear clusters y sistemas regionales de innovación por decreto o por medio de una ley, se debe invertir en recursos humanos, centros de investigación e instituciones de apoyo, y vincular las capacidades de las universidades en cada región con la demanda de las empresas."

Es aquí donde relacionamos la situación de la falta de personal calificado la cual podrían ser beneficiados con la implementación de mayores centros de investigación, y también donde la educación superior universitaria se vea beneficiada e involucrada con incentivos que desarrollen de mejor manera su capacidad de formación a las necesidades del mercado y las demandas de la sociedad.

Lo que se busca es que la formación futuros científicos en nuestro país se relacione con las necesidades y problemas reales que se presenten, y que y que ta,mbién se orienten a la formación de personas calificadas no solo parea desenvolverse en el mundo del trabajo, sino que también sean capaces de generar conocimiento aplicado a la realidad.

Vela, Acevedo, Yesquen y Venturra (2018: 147) afirman que "en el Perú el objetivo primordial y reto pendiente en cuanto a la ciencia y tecnología hace referencia a fortalecer las capacidades científicas apuntalando a un cambio estructural de la matriz productiva peruana, basando el crecimiento de la economía peruana en el conocimiento, requiriendo una inversión constante y del desafío del incremento de la inversión al 2021 del 0.7\% del PBI en I\&D, generando de esta manera un ecosistema innovador con alto potencial de 
crecimiento; cuyo resultado final será el incremento de la competitividad del país (Presidencia del Consejo de Ministros, 2016, p. 144-148)."

Por lo que se ve se necesita fortalecer una política de estado en que los aspectos científicos y tecnológicos sean claves para el desarrollo económico peruano y permitan un cambio estructural del mercado peruano, llámese la minería en primer lugar y en segundo los sectores de pesquería, agrícolas, comerciales, entre otros. Es en estos ámbitos donde también se hace presente la falta de personal peruano calificado por lo que las empresas deciden traer a sus especialistas extranjeros a cubrir puestos relacionados a la investigación y la innovación.

Inexistente cultura de investigación

Para Vela, Acevedo, Yesquen y Venturra (2018:146), "los resultados de investigación y desarrollo tecnológico que se obtienen están desalineadas de las necesidades del país y más aún del desarrollo de los departamentos del interior del país, es decir se realizan investigaciones que no responden a las necesidades sociales, económicas y ambientales." Si bien las investigaciones e innovaciones se llevan a cabo como parte del desarrollo del país, no son reconocidas ni valoradas por la sociedad peruana, y es por ello que no tienen relación alguna con cubrir las necesidades de la población. Esto pasa desapercibido por la mayoría de nuestra población por la escasa cultura científica que existe en el Perú. Nuestros jóvenes en su etapa escolar no logran relacionarse con temas científicos y tecnológicos, agregando aún más las serias deficiencias de sus profesores en estas áreas. Cosa similar parece ocurrir en la formación profesional de administración en la mayoría de universidades de nuestro país.

Al no existir una cultura científica es complicado que los jóvenes puedan seguir una orientación adecuada en el proceso de elegir una carrera profesional y en desarrollarla sobra bases científicas y tecnológicas; aunque algunas instituciones privadas puedan aprovechar esta situación para lucrar negativamente.

Labor de CONCYTEC por promover la ciencia y tecnología

Líneas anteriores nos referimos a las insuficiencias, tanto económicas como las de capital humano, para el desarrollo de la ciencia y tecnología, pero para contrarrestar estas barreras es que entra a tallar el CONCYTEC, una institución rectora del Sistema Nacional de Ciencia y Tecnología e Innovación Tecnológica, por su siglas SINACYT, con la finalidad de normar, dirigir, orientar, fomentar, coordinar, supervisar y evaluar las acciones del Estado en el ámbito de la Ciencia, Tecnología e Innovación Tecnológica. 
En uno de sus últimos recientes ejemplares, Concytec (2016:5) nos habla de "la introducción y promoción de la innovación tecnológica aporta al desarrollo humano el aumento de la productividad y el crecimiento económico del país, lo que a su vez genera mayores recursos para la inversión en educación, salud, comunicaciones y empleo, posibilitando el desarrollo de las capacidades humanas."

La innovación tecnológica no solo es una muestra del talento humano con la herramientas y capital necesario, también conforma parte del crecimiento económico de nuestro país como una partida directa para una acto cíclico si es gestionado de la manera adecuada y por personas calificadas. El resultado serán las inversiones en los sectores de mayor problemática con efectos igualitarios a países que tiene como política el desarrollo en los campos de la ciencia y tecnología.

Según Concytec (2016), "en la actualidad hay pleno consenso de que la ciencia, tecnología e innovación tecnológica tiene un impacto positivo en las tasas de crecimiento económico (...) Dentro de estos indicadores, la inversión en investigación y desarrollo es uno de los más emblemáticos, ya que indica el esfuerzo que hacen los países para generar, en forma sistemática, nuevos conocimientos en la economía." (p. 5)

Comprendamos que la reestructuración e inversión en la innovación tecnológica no son exclusivos solo de estudios y prácticas netamente científicas como es bien comprendido por el común de la población, la economía mundial y los sistemas de control son parte del impacto positivo del trabajo continuo en la ciencia y tecnología por parte de los gobiernos de turno para otorgar resultados de crecimientos económicos de igual manejo a las potencias mundiales.

Política nacional para el desarrollo de la ciencia y tecnología

Concytec (2016) expresa que "diversos países han implementado políticas de fortalecimiento de la infraestructura tecnológica, en virtud al rápido cambio y avance tecnológico. En el caso de China, cuenta con el Programa de Infraestructura Nacional de Ciencia y Tecnología, cuyo objetivo es fortalecer las capacidades de investigación en los diferentes niveles (básico, aplicado y desarrollo).” (p. 11)

Que tanto puede generar la implementación de una política nacional? China es un claro ejemplo que aun siendo una potencia económica continua trabajando en el fortalecimiento de las capacidades humanas. Son ellos los que generan la evolución constante de la innovación tecnológica para no competir con el resto, si no competir con ellos mismos, buscan superarse y obtener mejores resultados. 
Concytec (2016) también refiere "el Reino Unido tiene una política de financiamiento de laboratorios de clase mundial, así como acceso a este tipo de laboratorios en otras partes del mundo, en el convencimiento que es un factor importante para tener resultados sobresalientes en su productividad académica y en los resultados en innovación.” (p. 12) Vemos la contraparte de lo que vendría realizando China con la innovación tecnológica, donde el Reino Unido con el poder económico financia centros de investigación de talla mundial. El balance del financiamiento y la capacidad de otorgar una educación fortalecida otorgan efectos en cuanto a crecimiento y productividad en un país.

En nuestra realidad, Concytec (2016) expresa: "En ese sentido, se hace necesario contar con una masa crítica de investigadores y técnicos calificados, para lo cual se hace evidente la importancia de contar con programas de pregrado y postgrado de calidad, e institutos tecnológicos con infraestructura y docentes adecuados." (p. 11)

Al igual que en China o Reino Unido, un política nacional para el desarrollo de la Ciencia y Tecnología en nuestro país debe basarse en la implementación de programas con enfoque igualitario para todo estudiante, con acceso por igual a las herramientas e información sin barreras burocráticas si es que buscamos fortalecer las capacidades investigativas e innovadoras. Todo ello en conjunto con los docentes calificados, las infraestructuras correctas y generando la competencia interna para trabajar en el desarrollo potencial de los estudiantes.

Supervisar y evaluar las acciones del Estado son los pilares finales y claves de Concytec para poner en marcha el desarrollo de la Ciencia y Tecnología en el Perú, una institución con un enfoque de carácter globalizado para el entendimiento de nuestra realidad en comparación países con políticas establecidas y resultados agradables.

El reto del Desarrollo Nacional a partir del sistema de Ciencia y Tecnología

Las oportunidades para nuestro país son diversos, y aunque dependemos de inversiones extranjeras, podemos obtener un enfoque por otro camino logramos crear un sistema científico y tecnológico para seguir trabajando en el Desarrollo Nacional.

Una vez más, Concytec (2016) refiere que "la generación del conocimiento por medio de la investigación y desarrollo produce externalidades positivas en la sociedad, ya sea por el uso directo de tecnologías tales como como el Internet que ha permitido vencer barreras geográficas y aumentar la comunicación entre las personas o por los nuevos procedimientos diagnósticos que permiten detectar enfermedades en una etapa temprana." (p. 10) 
Para lograr el eficiente Desarrollo Nacional debemos recurría a la más grande tecnología de los últimos años: El Internet, una infinita base de datos con información, si bien no del todo autenticada, de fácil acceso, a un costo moderado y sin barreras existenciales en la que actualmente es pilar de la vida cotidiana. Gracias a inversiones extranjeras en conjunto con la gestión pública, existen programas para el acceso de la educación en las zonas de mayor índice de analfabetismo y únicamente con la implementación de esta tecnología, un vasto ejemplo cuando una herramienta es del todo productiva si se logra ver desde esa perspectiva.

Objetivos de la Política Nacional

De acuerdo con Seclén (2017), “...La investigación básica debería orientarse hacia el conocimiento, conservación y uso racional de nuestra gran diversidad de recursos naturales, ecosistemas, fuentes de energía, bosques, entre otros temas, ya que nos hacen falta estudios adecuados sobre estas diversidades que no estamos aprovechando." (Franciso

Sagasti, 2017, p.134)

Somos un país privilegiado geográficamente pero no sabemos aprovechar esa oportunidad, mucho menos los recursos naturales que son explotados por los capitalistas extranjeros con facilidades políticas. Solo en nuestra capital podemos ser testigos del nefasto índice de contaminación y el inadecuado consumo de servicios de primera necesidad, es por ello que las futuras investigaciones deberían centrarse en la conservación y el proceso productivo inteligente de recursos renovables.

Para Seclén (2017), “... Considero que las políticas de innovación deberían promover principalmente la invención y la innovación tecnológica, ya que estas conllevan mayor riesgo, tienen gran impacto, son rentables y requieren de personal altamente capacitado." (Franciso Sagasti, 2017, p.135-136)

Curiosamente como otros países, generar Programas de Innovación Tecnológica en conjunto con la innovación de nuevas herramientas que solo son demandadas por las necesidades del mercado, generando una mayor rentabilidad sin malgastar el poco financiamiento con el que se cuenta. Al ser una tecnología rentable y con un solo propósito, tendrá un gran impacto en su puesta en marcha, más aun si se cuenta con el personal calificado que estuvo en todo el proceso de elaboración.

Vela, Acevedo, Yesquen, Venturra (2018) creen que "los problemas que deben enfrentar los emprendedores van desde la presencia de barreras burocráticas, pasando por reducida 
capacidad para gestionar negocios y actividades tecnológicas, y reducida oferta de servicios relacionados." (p. 146)

Nuestra razón de ser en cuanto a la economía nacional recae en el poder de los emprendedores, pero como poder tener un monitoreo continuo de su crecimiento en con la existencias de normativas que dificultan la formalidad de los empresarios y solo benefician la desfachatez de los grandes capitalistas quienes al tener poder pueden acceder a la tecnología. Los Programas de capacitación en gestión administrativa, en conjunto con el desarrollo de la ciencia y tecnología, pueden coexistir para el benefício propio del país si dejamos a un lado los intereses personales y logramos pensar en el futuro.

Constitución de un Ministerio de Ciencia, Tecnología e Innovación

De acuerdo con Morris (2019) "cada ministerio e institución relacionadas a la ciencia, tecnología e innovación no debe trabajar en forma separada, sino que debe existir una institución responsable de dar las políticas públicas, leyes, y otras disposiciones para el desarrollo de la ciencia y la tecnología de una manera planificada, integrada para el desarrollo del país hacia una sociedad de la información y del conocimiento como lo están haciendo otros países."

La creación de una única institución seria la clave para la ejecución de las políticas relacionadas con la ciencia y tecnología. Dicha entidad no sería otra que un Ministerio de Ciencia, Tecnología e Innovación, un modelo que no es ajeno en nuestra región puesto que países como Colombia, Chile, Brasil y Costa Rica ya cuentan con este tipo de ministerio que logra gestionar de manera eficiente los recursos, demostrando a la población que existe una visión integral de crecimiento y desarrollo, a largo plazo. Al mismo tiempo, se fomentaría el desarrollo empresarial y la formación de un gobierno digital, sin papeles, con trámites rápidos y más transparentes. Una solución inmediata a los agónicos procesos que son el pesar de cada día en nuestra población al asistir a una entidad estatal.

Conclusiones

El Perú presenta grandes retos si es que tomamos nuestra actualidad como punto de partida para el desarrollo de la ciencia y tecnología, desde la insuficiente inversión económica en estos campos como base para el fortalecimiento de los estudiantes en una etapa temprana de formación académica, seguido de la importancia estructural de centros especializados con la tecnología no suficiente, solo necesaria acorde con las necesidades de investigación. 
Es clave de los cimientos de la educación escolarizada contar con profesionales capacitados en investigación, para generar en autoridades, profesores y estudiantes una cultura científica que, además, se exprese en la aplicación de métodos de enseñanza y aprendizaje que favorezcan la investigación como medio de construir conocimientos. Es así como con el proceso se despertaría las habilidades humanas junto a los componentes de innovación para producir tecnología.

Un paso importante en el proceso de crear esa cultura investigativa en la Facultad de Ciencias Administrativas y Recursos humanos constituye la inclusión en todas las asignaturas la exigencia de practicar la investigación formativa como un componente de nuestra formación profesional y el poner a nuestro alcance la respectiva Guía para Desarrollar la Investigación Formativa.

Por último, se quiere insistir que el desarrollo de la ciencia y tecnología pueda dar un paso más allá en cuanto a planificación y, en conjunto, con capital privado con enfoque en la concienciación de los problemas que enfrenta nuestro país, referidos al desarrollo sostenible, desarrollo humano, biodiversidad y potencialidades económicas, formación del talento, entre otros aspectos, que faciliten la ejecución de proyectos de solución y forme parte del desarrollo humano, con la continua búsqueda del desarrollo sostenible tras la ejecución de políticas públicas gestionadas por un Ministerio de Ciencia, Tecnología e Innovación.

Referencias

Bunge, M. (1985). Seudociencia e ideología. México: Alianza Editorial.

Bunge, M. (2014). La ciencia, su método y su filosofía. Editorial Laetoli, (5), 24-45.

CONCYTEC (2016). Crear para Crecer, Política Nacional para el Desarrollo de la Ciencia, Tecnología e Innovación Tecnológica. Lima: Consejo Nacional de Ciencia, Tecnología e Innovación. 5-27. Recuperado de: https://portal.concytec.gob.pe/images/documentos/Politica_Nacional_CTI2016.pdf Morris, E. (17 de enero 2019). La necesidad de contar con un Ministerio de Ciencia, Tecnología e Innovación. Conexiónesan. Recuperado de https://www.esan.edu.pe/conexion/actualidad/2019/01/17/la-necesidad-de-contarconun-ministerio-de-ciencia-tecnologia-e-innovacion/

Seclén, J. P. (2017). Políticas de ciencia, tecnología e innovación en el Perú. Entrevista a Francisco Sagasti. Revista de Ciencias de la Gestión(2), 135-136. Recuperado de: http://revistas.pucp.edu.pe/index.php/360gestion/article/view/19055/0

Vela, Acevedo, Yesquen, Venturra et al. (2018). Ciencia, Tecnología e Innovación en el 
Perú. Necesidad de una Política Pública Descentralista, que Institucionaliza las

Alianzas Academia-Empresa-Estado y Sociedad Civil GeoGraphos, Revista Digital para

Estudiantes de Ciencias Sociales. Vol. 9 (106), 138-157. Recuperado de:

https://rua.ua.es/dspace/bitstream/10045/75207/1/Lindon_Vela.pdf 